\title{
Achilles Tendon Rupture: Double the Trouble
}

\author{
Wafiuddin Ahmad¹, Ahmad Faizal Roslan¹, Faisal Amir², Khairul Nizam Siron² \\ ${ }^{1}$ Department of Surgical-Based, Faculty of Medicine and Health Sciences, Universiti \\ Malaysia Sabah \\ 2Department of Orthopedic, Traumatology and Rehabilitation, Kulliyah of Medicine, \\ International Islamic University Malaysia.
}

Introduction: Achilles tendon is the strongest tendon in the body . Achilles tendon rupture is a debilitating ankle injury especially among the athletes. We would like to highlight an unusual case of acute dual-level injuries of the Achilles tendon. Case report: A 30-year-old footballer presented with left ankle injury during a football tournament. Examination revealed tenderness at posterior left heel, palpable gap at Achilles tendon region and positive Thompson test. Radiological assessment showed dual-level injuries of the Achilles tendon-proximally was a rupture at musculotendinous junction and distally was an avulsion calcaneal fracture. We performed a mini-open approach Achilles tendon reconstruction for this patient. First, we reconstructed the distal avulsion calcaneal fracture using double row anchor sutures technique. Next, we repaired the proximal ruptured Achilles tendon using percutaneous Achilles reconstruction system (PARS) from Arthrex. Postoperatively, patient was put on functional rehabilitation protocol. At present, patient recovers well and regains back the Achilles tendon function. In conclusion, segmental Achilles tendon injury is rare and its surgical treatment can be challenging and must be well-planned. 